\title{
Application Of Artificial Neural Networks For Path Loss Prediction In Railway Environments
}

\author{
Di Wu, Gang Zhu, Bo Ai \\ State Key Laboratory of Rail Traffic Control and Safety \\ Beijing Jiaotong University \\ Beijing, China \\ e-mail: 09111054@bjtu.edu.cn
}

\begin{abstract}
To balance the precision and generality of the prediction model, a new path loss artificial neural network (ANN) prediction model for railway environments is presented firstly in this paper. The utilization of back propagation ANN can overcome some disadvantages of such conventional prediction models as empirical and deterministic models. The training data is based on the electric field strength measurements in the Zhengzhou-Xi'an express railway line in China. Through many attempts and comparisons, the suitable architecture and learning algorithm are chosen in the proposed model. After training, the proposed model can predict the path losses accurately in typical similar railway environments. Comparisons between a conventional model and the proposed model, with the measured and predicted data show that the proposed model is sufficiently applicable in railway scenarios.
\end{abstract}

Keywords-Artificial neural network (ANN);back propagation network (BPN); learning algorithm; path loss;railway.

\section{INTRODUCTION}

A well-suited propagation model is very important for planning wireless networks. An accurate estimation of path loss provides a basis for proper determination of the field strength, signal-to-noise ratio, carrier-to-interference ratio, etc. However, to predict the propagation path loss is a difficult and complex task. The conventional prediction method can generally be classified into two categories: empirical and deterministic models. Empirical models [1-3], which are derived from measurements, have satisfying computational efficiency. But field works are very cumbersome and tedious. In addiction, empirical models are not very specific, which can not be used in different environments without modification. On the other hand, deterministic models, based on the ray tracing technology $[4,5]$, suffer from the excessive computational time and need detailed environmental information, although they are more site-specific.

ANN has been successfully used in several applications such as interpolation, data clustering, pattern recognition and forecasting $[6,7]$. The ability to solve nonlinear function approximation problems is required for path loss predictions. As an alternative method, artificial neural network (ANN) model can achieve balance between the precision and generality. Another key feature of ANN is the intrinsic parallelism allowing for a fast evaluation of solutions. Field

This work was supported by the Joint Program of the National Natural Science Foundation of China under Grant number 60830001, Key Project of State Key Lab. of Rail Traffic and Control under Grant number RCS2008ZZ007 and RCS2008ZZ006, and the Program for Changjiang Scholars and Innovative Research Team in University under Grant No. IRT0949. strength prediction models using ANN have been proposed for both outdoor and indoor environments [8-13]. They used corresponding network architectures in terms of the specific scenarios in public networks. A large number of measurement results verified the effectiveness of the ANN model. However, none of them has taken investigation on such dedicated networks, such as railway environments.

Path losses in railway environments are different from the path loss in public networks because the characteristics are totally different. For example, public networks often consist of urban, hilly scenarios and so on. However, the scenarios in railway environment ordinarily include viaduct, cutting and plain. More flat-level environments are needed for safe and fast moving, which leads to the differences in the main propagation mechanisms from the public network environments. Therefore, the conventional prediction methods are not applicable under such conditions any more. In this paper, a new ANN model for the railway environments is proposed firstly. And the suitable ANN structure and parameters are chosen in terms of the practical railway environments. Some comparisons between conventional methods and the proposed method are made to show the superiority of the ANN model. Measurements are used to verify the effectiveness of the proposed model.

The remainder of this paper is arranged as follows. Section II shows how to set up the field measurements. Section III provides a brief overview of the architecture of the proposed ANN model and a description of the realization process. Then the back propagation ANN models trained by different algorithms are compared to find the suitable ANN model for typical railway environments. In section IV, the path losses predicted by the proposed model are compared with the predictions by a conventional model. Then comparison between the measured data and the ANN-based prediction is described. In section $\mathrm{V}$, we discuss the conclusion and future work.

\section{MEASUREMENT DESCRIPTION}

To train the ANN and validate the predicted results, some field work is required. The data is based on the measured results obtained from the Zhengzhou-Xi'an express railway line. Viaducts, cuttings and plains are typical scenarios in railway environments. The path loss measurement was carried out based on the received field strength on the receivers, which 
is sent from the base station. The base stations are placed every $3 \mathrm{~km}$ or so. During the measurements, a radio-frequency carrier was radiated by a vertically polarized omni-directional antenna. The carrier frequency was set at the order of $930 \mathrm{MHz}$ in this paper. The receiving antenna was mounted on the top of the train. The received field strength was measured using the Griffin software and global position system. To deal with a great number of data, the data was delivered to the computer. The fast fading was eliminated by the moving-average method. The final results were achieved by the software exploited by Beijing Jiaotong University. Then the obtained data set in each railway section line was split into two sets. The first set (training set) was used to network training. The second set (validation set) was used to evaluate the performance of the learned network.

The measured path loss can be computed by

$$
L p=P_{T}+G_{T}+G_{R}-L_{f}-L_{i}-P_{R},
$$

where $P_{T}$ and $P_{R}$ is the transmitted power and the received power, respectively, $G_{T}$ and $G_{T}$ is the gain of the transmitting antenna and the receiving antenna, respectively, $L_{f}$ is the loss of the feeder line, and $L_{i}$ is the insertion loss. The related parameters for measurements are as follows: the transmitted power is $43 \mathrm{dBm}$ and the received power can be obtained from the receiver. The gain of the transmitting antenna and the receiving antenna is $17 \mathrm{dBi}$. The loss of the feeder line is about $3.5 \mathrm{~dB}$. The insertion loss is about $3.3 \mathrm{~dB}$.

\section{ARCHITECTURE OF ANN MODEL}

\section{A. Description of $A N N$}

The ANN models contains many types, like back propagation, radial basis function, etc. They have been widely used in different areas, including pattern recognition, image processing, forecasting, automatic control and so on. The ANN model, proposed in this paper, is based on back propagation network (BPN) architecture. Due to the sigmoid activation functions, the model has shown good performances in solving problems with mild nonlinearities on the set of noisy data. This case fully corresponds to the field strength prediction.

The architecture of a typical BPN [14] consists of a sequence of three or more successive layers or groups of basic units called neurons, nodes, or processing elements, which are fully or partly interconnected to other processing elements in the same or different layers multiplied by connection weights and added by threshold values. The first layer is the input layer where input data is sent to the network for processing. The last layer is the output layer which is the response of the learned network. The others are called hidden layers. The rough architecture of BPN is shown in Figure 1 [10].

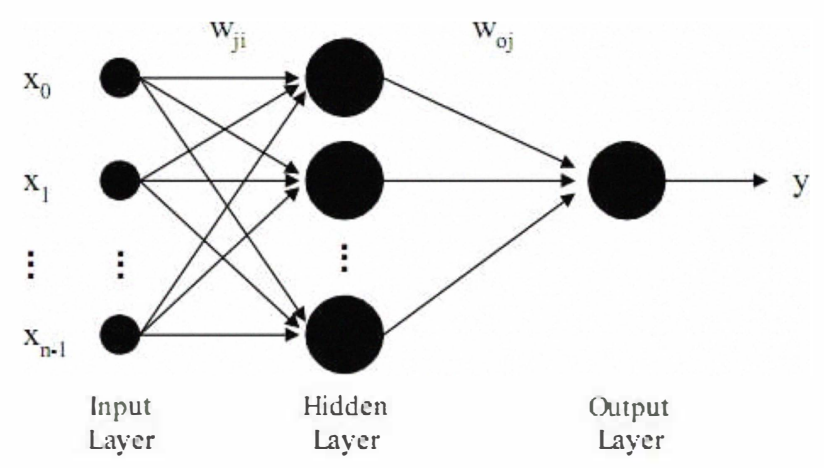

Figure 1. The configuration of the BPN

The output of the neural network is described by the following equation:

$$
y=F_{0}\left\{\sum_{j=0}^{M} w_{o j}\left(F_{h}\left(\sum_{i=0}^{N} w_{j i} x_{i}\right)\right)\right\},
$$

where $x_{i}$ is the $i^{\text {th }}$ element of the input vector, $F_{0}\{\bullet\}$ is the activation function of the second layer, $F_{h}(\bullet)$ is the activation function of the first layer, $w_{o j}$ represents the synaptic weights from neuron $\mathrm{j}$ in the hidden layer to the single output neuron, $w_{j i}$ is the connection weights between the neurons of the hidden layer and the inputs, $\mathrm{N}$ and $\mathrm{M}$ is the number of the input neuron and hidden neuron, respectively.

The systematic parameters, such as weight values, threshold values, are adjusted by the learning process. This learning phase of the network performs based on the mean squared error E between predicted and measured path loss for a set of appropriately selected training examples, which is given by:

$$
E=\frac{1}{2} \sum_{i=1}^{m}\left(y_{i}-d_{i}\right)^{2},
$$

where $y_{i}$ is the output value calculated by the network and $d_{i}$ represents the expected output, i.e., the measured value. Based on the sigmoid and linear function, the prediction curve may be close to any nonlinear curve in theory.

But this type of ANN model also has some limitations [14]. Firstly, the convergence of the training process is relative slow due to the requirement of the stability. Secondly, the selection of the learning rate is important because the prediction process will be instability if the learning rate is chosen too large. And the prediction time will be too long if the learning rate is too small. Finally, the problem may have no solutions even though it is obtainable in theory. Therefore, the selection of the input, learning rate and the training pattern plays an important role. They will be discussed below, respectively.

\section{B. ANN Model Design}

It should be noted that the selections of the input, output, the number of the layers, weight values, threshold values and 
the learning algorithms are important. Otherwise the predicted results will be affected. In this model, the distance between base station and receiver is selected as the input units of the neural network. And the output node of the neural network is the received signal strength. Input and output parameters are normalized so that they take values in the range $[0,1]$. This is to avoid the permanent saturation of the units in the network at the very beginning of the learning and make it insensitive to the learning process [13]. Normalization is taken from premnmx function in matlab, which is given by:

$$
P_{n}=2 \times\left(P-P_{\min }\right) /\left(P_{\max }-P_{\min }\right)-1,
$$

where $P_{\eta}$ is the value after normalization, $\mathrm{P}$ is the input vector, $P_{\min }$ and $P_{\max }$ is the maximum and the minimum value among the vector $P$, respectively. By many attempts and comparisons, ten hidden neurons, three-layer ANN model with the combination of sigmoid and linear activation function were chosen in this paper.

In order to make the training time as short as possible, the learning parameters, like weight values and threshold values, were selected in a default way. As a learning rule, error back propagation is chosen since it provides a computationally efficient method for changing the weights in a back propagation network [15].

For the learning algorithm selection, there are many methods to improve the learning process, such as scaled conjugate gradient (SCG) algorithm, Fletcher-Reeves conjugate gradient (FCG) algorithm, quasi-Newton method (QN), Levenberg-Marquardt (LM) algorithm and Powell-Beale conjugate gradient (PCG) algorithm [14]. These algorithms aim to improve the performance and enhance the convergence speed. However, every algorithm has its own advantages and disadvantages. Whether it is suitable depends on the actual application situations. In order to select a suitable learning algorithm, comparisons among the ANN predicted results with these algorithms are discussed here.

TABLE I. ERror Statistics For Path Loss Prediction by ANN WITH DIFFERENT ALGORITHMS

\begin{tabular}{|c|c|c|}
\hline Algorithm & Mean $(\mathrm{dB})$ & Variance $(\mathrm{dB})$ \\
\hline SCG & 1.19 & 0.95 \\
\hline FCG & 1.20 & 0.95 \\
\hline QN & 1.11 & 0.90 \\
\hline LM & 1.10 & 0.80 \\
\hline PCG & 1.16 & 0.92 \\
\hline
\end{tabular}

The prediction is completed with the help of the neural network toolboxes of Matlab 7.0 [16]. The first set of the measurement data in certain scenario is used to train the ANN model, and the rest of them are chosen to validate the trained model. TABLE I illustrates the error statistics for ANN model with different algorithms. The mean and variance is the error mean and error variance between the measured data and the predicted data by ANN model, respectively. The measurements were made in a viaduct scenario in some railway section line. The relative height of the transmitting antenna was $20-40 \mathrm{~m}$ and the height of the receiving antenna was about $3.5 \mathrm{~m}$. The frequency used here was $932.8 \mathrm{MHz}$. The results show that the ANN with LM algorithm has the better performance in this case. Because the best features of the Gauss-Newton method and the steepest-descent method are combined in this algorithm, it avoids many other limitations and has a fast convergence [17]. Therefore, the ANN with LM algorithm is used in the following prediction $\mathrm{s}$.

\section{COMPARISONS}

\section{A. Comparison Between Proposed Model and Hata Model in Specific Areas}

After deciding the ANN architecture, the proposed model is applied to practical situations. Three typical scenarios in railway environments are discussed. Because a widely used empirical model in public network planning is the Hata model with corrections, the Hata model is chosen for comparison. Meanwhile, the free space loss is used as a baseline in Figure 2.

As a first step, the well known formula of Hata, which represents an empirical approximation for path loss Lp in open areas, is given by [2]:

$$
\begin{aligned}
L p(d B)= & 69.55+26.16 \log f_{c}-13.82 \log h_{b}-a\left(h_{m}\right) \\
& +\left(44.9-6.55 \log h_{b}\right) \log d-4.78\left(\log f_{c}\right)^{2} \\
& +18.33 \log f_{c}-40.94,
\end{aligned}
$$

where $f_{c}$ is the frequency $(\mathrm{MHz})$ with the range from 150 $\mathrm{MHz}$ to $1500 \mathrm{MHz}, h_{b}(\mathrm{~m})$ is the relative height of the transmitting antenna in the base station, $h_{m}(\mathrm{~m})$ is the height of the receiver, $d(\mathrm{~km})$ is the distance between the base station and the receiver and

$a\left(h_{m}\right)=3.2\left(\log 11.75 h_{m}\right)^{2}-4.97$ for $f_{c} \geq 300 M H z$ in a large city. Other situation can be modified using corrections in the corresponding scenarios [2]. Then the predicted path losses in three typical scenarios are analyzed below.

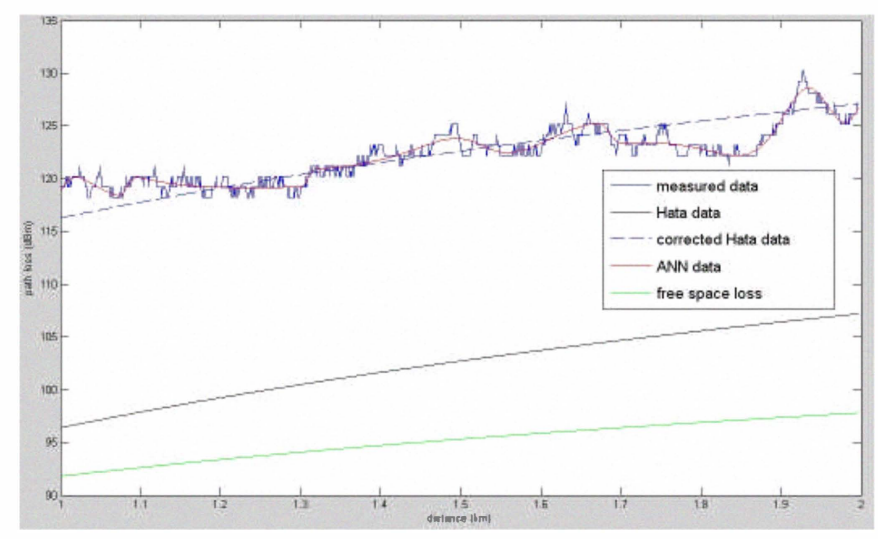

(a) Viaduct scenario 


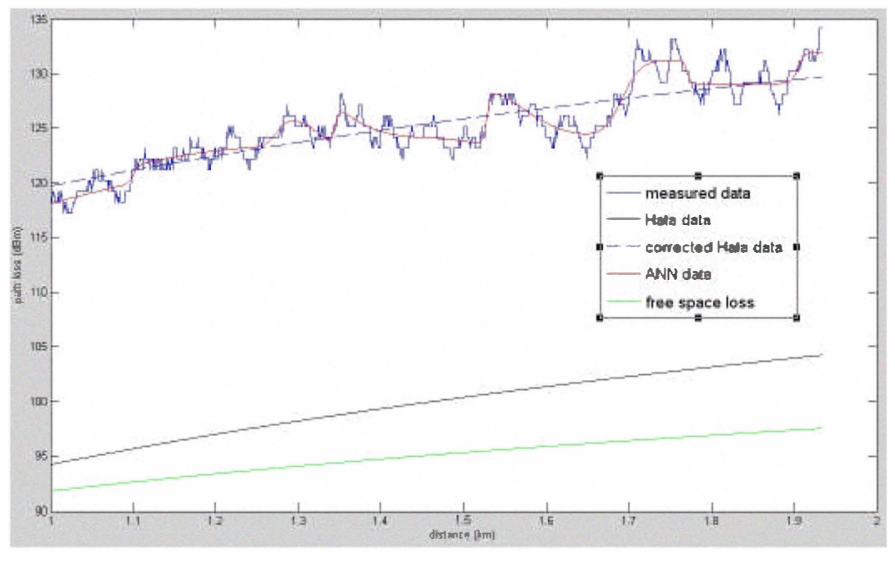

(b) Cutting scenario

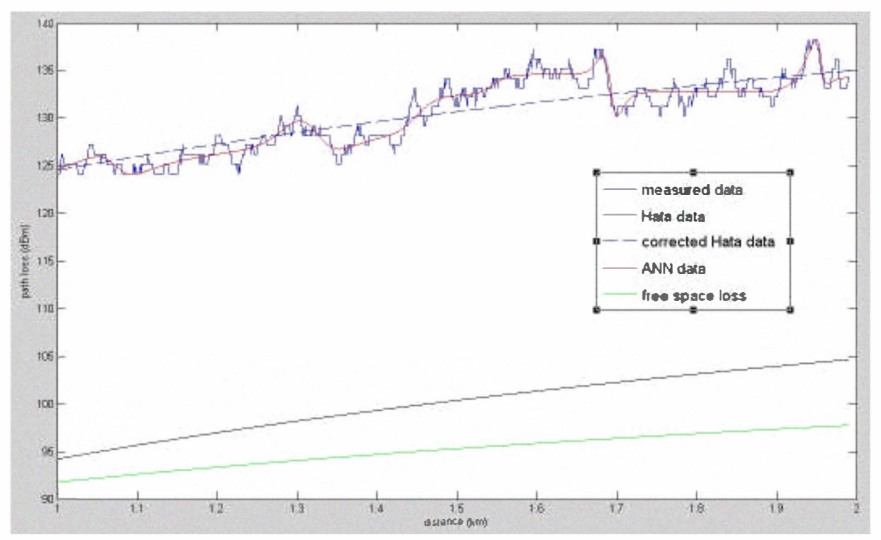

(c) Plain scenario

Figure 2. Measured and predicted path loss in different scenarios

Figure 2 shows the comparisons among the measured path losses, the path losses predicted by the proposed model and the path losses predicted by Hata model without corrections in three typical railway environments. During the measurements, the height of the receiver was $3.5 \mathrm{~m}$. The distance range chosen in the comparisons is about $1-2 \mathrm{~km}$. Because if the distance is less than $1 \mathrm{~km}$ at the frequency of about $900 \mathrm{MHz}$, there exists the near-filed effect, which will affect the prediction [2]. And for the distance less than $1 \mathrm{~km}$ does not affect the network planning, this range is not required to be considered in this case. And the distance more than $2 \mathrm{~km}$ is overlapped with the coverage of another base station. Therefore, just 1-2 km distance was taken into account. In terms of the practical measurement situations, different frequencies and the relative height of the transmitting antenna were used in different railway sections, which are list in TABLE II.
TABLE II. ERROR STATISTICS FOR PATH LOSS BY HATAAND ANN IN DIFFERENT SCENARIOS

\begin{tabular}{|c|c|c|c|c|c|}
\hline \multicolumn{2}{|c|}{$\begin{array}{l}\text { prediction } \\
\text { model }\end{array}$} & $\begin{array}{c}\text { Frequency } \\
(\mathrm{MHz})\end{array}$ & Height (m) & Mean $(\mathrm{dB})$ & $\begin{array}{c}\text { Variance } \\
(\mathrm{dB})\end{array}$ \\
\hline \multirow{3}{*}{ viaduct } & Hata & \multirow{3}{*}{932.8} & \multirow{3}{*}{23} & 19.9 & 2. 9 \\
\hline & $\begin{array}{c}\text { corrected } \\
\text { Hata }\end{array}$ & & & 1. 3 & 1.1 \\
\hline & ANN & & & 0.6 & 0.2 \\
\hline \multirow{3}{*}{ cutting } & Hata & \multirow{3}{*}{932.4} & \multirow{3}{*}{33} & 25.5 & 3.2 \\
\hline & $\begin{array}{c}\text { corrected } \\
\text { Hata }\end{array}$ & & & 1.4 & 1. 1 \\
\hline & ANN & & & 0.8 & 0.4 \\
\hline \multirow{3}{*}{ plain } & Hata & \multirow{3}{*}{932} & \multirow{3}{*}{33} & 30.3 & 3.5 \\
\hline & $\begin{array}{c}\text { corrected } \\
\text { Hata }\end{array}$ & & & 1.5 & 1.1 \\
\hline & ANN & & & 0.8 & 0.3 \\
\hline
\end{tabular}

In TABLE II, the corrected Hata data is the value computed by (5) added by the error mean between Hata predicted data and the corresponding measurements. From TABLE II and Figure 2, the data from the Hata model deviates from the measured data largely. The worst mean deviation is up to 30.3 $\mathrm{dB}$. It is indicated that the Hata model can not be applicable in this type of environment. However, after corrections, the corrected Hata data is still worse than the data predicted by ANN model. As could be expected, the ANN learns better and faster from the simple set of data. For the ANN model, the maximum mean value is $0.8 \mathrm{~dB}$ and the maximum variance is $0.4 \mathrm{~dB}$. The results show an improvement in path loss prediction with the proposed model over the Hata model, even the corrected Hata predictions. Therefore, the proposed ANN model can predict the path loss accurately just trained by a small set of the measured data.

\section{B. Comparison Between Measurements and Predictions By Using the Trained ANN Model in Different Areas}

One of the advantages of the ANN model is the characteristic of generality. To show this potential advantage, the predictions by the trained ANN model were compared with the measurement in other railway section line with the similar scenario.

TABLE III. MEASURED AND PREDICTED PATHLOSS IN DIFFERENT SCENARIOS

\begin{tabular}{|c|c|c|}
\hline scenario & Mean $(\mathrm{dB})$ & Variance $(\mathrm{dB})$ \\
\hline Viaduct 1 & 1.73 & 1.70 \\
\hline Viaduct 2 & 1.82 & 1.5 \\
\hline Cutting 1 & 4.49 & 6.49 \\
\hline Cutting 2 & 3.24 & 7.03 \\
\hline Plain 1 & 2.92 & 2.31 \\
\hline Plain 2 & 2.39 & 3.96 \\
\hline
\end{tabular}




\section{ACKNOWLEDGMENT}

Railway sections

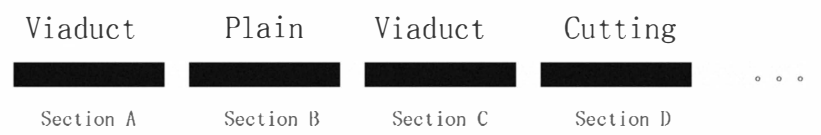

Figure 3. Layout sketch map of railway sections

In TABLE III, the mean and variance between measured data in one section line and the predicted data by the ANNbased model trained in another section line with the similar scenario are shown. That is, the measured data in section A is compared with the ANN-based results trained by the measured data in section $\mathrm{C}$ as shown in Figure 3. It should be noted that the scenario in section A is similar with the scenario in section C. From TABLE III, it is shown that the proposed model can predict the path loss well in the similar scenario. Although there are some differences between the path losses in two sections with similar scenario, the prediction effects are analogy. The maximum deviation occurred in cutting scenarios. This is because different cuttings have various slopes, which results in the differences in diffraction losses. Conclusively, the ANN-based model can predict the path loss for other similar scenario under certain precision, where no measured data is available. The predicted results show acceptable agreement with the measured data and validate the generality of the proposed model.

\section{CONCLUSION ANDFUTURE WORK}

The proper radio system design requires the accurate and reliable prediction model. And the path loss prediction is the most fundamental factor. To predict the path loss in railway environment as accurately and fast as possible, the proposed ANN model is introduced firstly in railway environment, through which some disadvantages of both empirical and deterministic propagation model can be overcome. Within the proposed ANN models, environment characteristics can be considered more easily than empirical models. On the other side, the ANN models are simple and computationally fast than deterministic models. The proposed ANN model is based on the popular back propagation network architecture. According to analyses, the suitable model architecture and learning algorithm are chosen for measurement environments. In comparison with other conventional model, more accurate predictions can be achieved using the proposed model after being trained by part of measured data. And comparisons between measurements and predictions by trained ANN model show that the proposed model can also be applicable in another railway section with the similar scenario. According to the results predicted by the proposed model, the prediction can be successfully used in railway environment with relatively high precision.

Due to the scope of this paper, the ANN model is just introduced in a simple way. To improve the performance and the generality of this type of model, more measurement parameters can be considered in the input vector. Of course, the fuzzy logic can also be incorporated into the ANN model.
We would like to thank to all the team that has conducted the measurements and delivered us the measured data in order to investigation a suitable and general propagation model.

\section{REFERENCES}

[1] Y. Okumura et al., "Field strength and its variability in VHF and UHF land-mobile services," Rev. Electr. Commun. Lab., vol. 16, pp. 825-837, Sept.-Oct. 1968

[2] M. Hata, "Empirical Formula for Propagation Loss in Land Mobile Radio Services," IEEE Trans. Vehic. Tech., vol. 29, no. 3, 1980

[3] "COST Action 231 - Digital mobile radio towards future generation systems", Final Report, European Communities, 1999

[4] S. Y. Seidel and T. S. Rappaport, "Site-specific propagation prediction for wireless in-building personal communication system design," IEEE Trans. Veh. Technol., vol. 43, pp. 879-891, Nov. 1994.

[5] G. Liang and H. L. Bertoni, "A new approach to 3-D ray tracing for propagation prediction in cities," IEEE Trans. Antennas Propagat., vol.46, pp. 853-863, June 1998.

[6] Abbasy, N.H.; El-Hassawy, W., "Power system state estimation: ANN application to bad data detection and identification", AFRICON, 1996., IEEE AFRICON $4^{\text {th }}$, pp. $611-615,1996$.

[7] Guangning Wu; Xiongwei Jiang; Hengkun Xie, "A neural network used for PD pattern recognition with genetic algorithm", Properties and Applications of Dielectric Materials, 2000. Proceedings of the 6th International Conference on, pp. 451 - 454. 2000.

[8] A. Neskovic, N. Neskovic, and D. Paunovic, "A Field Strength Prediction Model Based on Aritificial Neural Networks," Proc. 9 $9^{\text {th }}$ IEEE Med. Electro. Conf. -MELECON, Tel Aviv-Israel, May 1998.

[9] Ileana Popescu, et al. "ANN Prediction Models for Indoor Envrionment" Wireless and Mobile Computing, Networking and Communications, 2006. (WiMob'2006). IEEE International Conference on, pp. $366-371,2006$

[10] Huaibei Zhou, Fan Wang, Chunhua Yang, "Application of Artificial Neural Networks to the Prediction of Field Strength in Indoor Environment for Wireless LAN," Wireless Communications, Networking and Mobile Computing, 2005. Proceedings. 2005 International Conference on, pp. 1189-1192, 2005

[11] Ileana Popescu, et al. "APPLICATION OF GENERALIZED RBF-NN FOR PATH LOSS PREDICTION," Personal, Indoor and Mobile Radio Communications, 2002. The 13th IEEE International Symposium on, pp. 484-488, 2002.

[12] Ruben Fraile, Lorenzo Rubio, Narcis Cardona, "Application of RBF Neural Networks to the Prediction of Propagation Loss over Irregular Terrain," Vehicular Technology Conference, 2000. IEEE VTS-Fall VTC 2000. $52^{\text {nd }}$, pp. 878-884, 2000.

[13] A. Neskovic, N. Neskovic, and D. Paunovic, "Macrocell Electric Field Strength Prediction Model Based Upon Artificial Neural Networks", Selected Areas in Communications, IEEE Journal on, pp. $1170-1177$. 2002.

[14] Changhong Dong, "Matlab Neural Networks and Applications" (Chinese), National Defense Industry Press, 2007.9. pp. 77-92.

[15] D. E. Rumelhart, G. E. Hinton, and R. J. Williams, "Learning internal representations by error propagation," in Parallel Distributed Processing: Explorations in the Microstructure of Cognition,D.E. Rumelhart and J. L. McClelland, Eds. Cambridge, MA: MIT Press, 1986, pp. 318-362.

[16] H. Demuth, and M. Beale, Neural Network Toolbox For Use with MATLAB. User's Guide, Version 3.0

[17] Ivan Vilovic, Niksa Burum, Zvonimir Sipus, "A Comparison of Neural Network Models for Indoor Field Strength Prediction," ELMAR, 2007, pp. $251-254,2007$ 